\title{
Measurement of very-high-energy diffuse gamma-ray emission from Galactic plane with LHAASO-KM2A
}

\section{Rui Zhang, ${ }^{a, b, *}$ Shiping Zhao, ${ }^{a, c, *}$ Yi Zhang ${ }^{a}$ and Qiang Yuan ${ }^{a}$ on behalf of the LHAASO Collaboration \\ (a complete list of authors can be found at the end of the proceedings)}

\author{
${ }^{a}$ Key Laboratory of Dark Matter and Space Astronomy, Purple Mountain Observatory, Chinese Academy \\ of Sciences, \\ 210023 Nanjing, 9 Jiangsu, China \\ ${ }^{b}$ University of Science and Technology of China, \\ 230026 Hefei, Anhui, China \\ ${ }^{c}$ Institute of Frontier and Interdisciplinary Science, Shandong University, \\ 266237 Qingdao, Shandong, China \\ E-mail: zhaosp@mail.sdu.edu.cn, zhangrui@pmo.ac.cn
}

Galactic diffuse gamma ray emission (GDE) is introduced by the galactic cosmic rays interacting with the interstellar medium (ISM) and/or radiation fields (ISRF). Studying galactic diffuse TeV $\gamma$-ray emission would help to understand mechanisms of acceleration and propagation of galactic cosmic rays. LHAASO-KM2A with an large area of $1.36 \mathrm{~km}^{2}$, has an excellent ability to study VHE $\gamma$-ray astronomy and GDE. In this proceeding, method of background estimation and some techniques to reduce the contaminant of resolved $\gamma$-ray sources are briefly introduced. At last, we look into the inner galactic plane (IGP) and present the measurements.

$37^{\text {th }}$ International Cosmic Ray Conference (ICRC 2021)

July 12 th - 23rd, 2021

Online - Berlin, Germany

\footnotetext{
*Presenter
} 


\section{Introduction}

The Galactic diffuse $\gamma$-ray emission (GDE) is produced via the interactions between cosmic rays (CRs) and the interstellar medium (ISM) and/or radiation fields (ISRF). Typically there are three main components of the GDE [1], the decay of neutral pions produced by inelastic collisions between CR nuclei and the ISM, the inverse Compton scattering (ICS) of CR $e^{ \pm}$off the ISRF, and the bremsstrahlung radiation of $e^{ \pm}$in the ISM. The GDE is a very important probe of CR propagation and interaction. Different from the measurements of CR particles in the local vicinity, the GDE enables a direct measurement of CR distribution in the Milky Way, and can thus provide much more important information of the production and propagation of CRs.

\section{LHAASO Observations}

\subsection{The LHAASO Experiment}

The LHAASO experiment is a hybrid, large area, wide field-of-view observatory for CRs and $\gamma$-rays in a wide energy range which is under construction at Haizi Mountain $\left(100^{\circ} .01 \mathrm{E}, 29^{\circ} .35 \mathrm{~N}\right.$; $4400 \mathrm{~m}$ above the sea level), Daocheng, Sichuan province, China [2]. LHAASO serves as the most sensitive $\gamma$-ray detector for energies above a few tens of $\mathrm{TeV}$, and is expected to give revolutionary insights in the VHE domain of astroparticle physics, such as the origin and propagation of CRs, as well as the nature of VHE $\gamma$-ray sources. As the main part of LHAASO, square kilometers array (KM2A) has an area of $\sim 1.36 \mathrm{~km}^{2}$, consists of 5195 electromagnetic detectors (EDs) on the ground and 1171 muon detectors (MDs) underground.

\subsection{Mont Carlo Simulation}

To learn the efficiency of KM2A, Mont Carlo simulations based on CORSIKA and Geant4 packages are carried out. CORSIKA code (version7.6400) [3] is used to simulate cascade processes of primary particles in the atmosphere, with QGSII and GHEISHA for high and low energy hadronic interaction model, respectively. Directions of the simulation events are sampled isotropically with zenith angles ranging from $0^{\circ}$ to $70^{\circ}$. While the energies are sampled following a single power law with an index of -2 . At the end, the simulated spectral energy distribution (SED) is $2.55 \times 10^{-3} \mathrm{TeV}^{-1} \mathrm{~cm}^{-2} \mathrm{sr}^{-1}(\mathrm{E} / 1 \mathrm{TeV})^{-2}$.

The detector response of KM2A is simulated via a specific software G4KM2A [4], which is based on framework of the Geant4 package (v4.10.00) [5]. Using the same algorithms, both data and simulation events are reconstructed, thus the reliability of simulation can be verified. We find that the simulation is reliable based on the fact that distributions of several measurements like $N_{e}$ and $N_{\mu}$ are consistent with data[4], where $N_{\mu}$ is the number of muons collected by MDs and $N_{e}$ is the number of particles counted by EDs.

\subsection{Data}

In this work data collected from December 27, 2019 to November 30, 2020 are analyzed. For the consideration of a reliable reconstruction, only part of the data are accepted. The used criteria are the same as that in Ref.[6]. 
Showers introduced by $\gamma$ photons have less muons than introduced by CRs, leads to the different ratio of recorded $N_{e}$ to $N_{\mu}$. As adopted in Ref.[6], the same ratio definition is used to discriminate $\gamma$-rays and CRs,

$$
R=\log \left(\frac{N_{\mu}+0.0001}{N_{e}}\right)
$$

Fig. 1 shows survival ratios of $\gamma$-rays and CRs respectively.

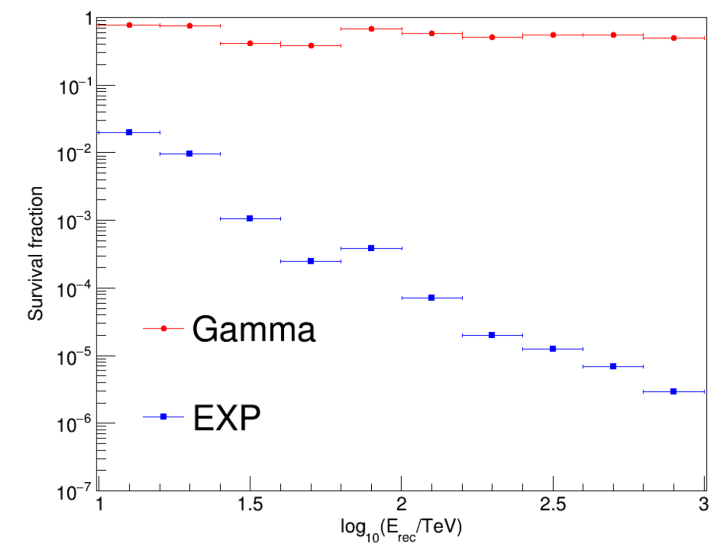

Figure 1: Surival fraction of gamma events and cosmic ray events

\section{Analysis}

\subsection{Background Estimation}

The map of the sky is binned into cells with a size of $0^{\circ} .1$ in both the right ascension (R.A.) and declination (Dec.) directions. Background is estimated through a modified direct-integral method. In this method, the distribution of the background is firstly figured out. Following this distribution, the background is sampled and counted.

After background estimation, the significance of an area can be evaluated by using a test statistic as two times of the logarithmic likelihood ratio, i.e., $T S=2 \ln \left(\mathcal{L}_{s+b} / \mathcal{L}_{b}\right)$, where $\mathcal{L}_{s+b}$ is the maximum likelihood for the signal plus background hypothesis and $\mathcal{L}_{b}$ is the likelihood for the background only hypothesis. According to Wilks's theorem [7], in our situation with only one free parameter, $\sqrt{T S}$ can be interpreted as the pretrial Gaussian significance.

\subsection{Forward-Folding SED Fitting Method}

We use a forward unfolding procedure to measure the SED of this source. Based on the simulation, the following $\chi^{2}$ equation is minimized to get the SED,

$$
\chi^{2}=\sum_{i=1}^{n}\left(\frac{\mathrm{N}_{\mathrm{s}}-\mathrm{N}_{\text {sim }}(\alpha, \beta)}{\sigma_{N_{s}}^{2}}\right)
$$

where $n$ represents number of energy bins, $\mathrm{N}_{\mathrm{s}}$ represents the excess of $\gamma$-rays at this bin, and $\mathrm{N}_{\text {sim }}(\alpha, \beta, \ldots)$ is evaluated by Mont-Carlo simulation with a given SED. The SED of GDE is assumed to follow a power-law spectrum $\frac{d N}{d E}=\phi_{0} \times\left(E / E_{0}\right)^{-\alpha}$, where $E_{0}=50 \mathrm{TeV}$ is a reference energy. 


\subsection{Influence of Resolved Sources}

Over TeV energy regime, the observed $\gamma$-ray flux is dominated by resolved sources. Thus resolved sources need to be extracted. Define the mask radius of a source $R_{M}$ as:

$$
R_{M}=M \cdot \sqrt{\sigma_{\mathrm{ext}}^{2}+\text { p.s.f. }}{ }^{2}
$$

where $\sigma_{\text {ext }}$ represents the extension of the source, and p.s.f. represents the variance of a 2-D Gaussian distribution fitting the point spread function at the first bin of energy, ranging from $10 \mathrm{TeV}$ to $15 \mathrm{TeV}$. Sources are extracted with a radius of $R_{M}$, in this proceeding, $M$ is assigned as 2 .

\section{Results}

We have measured $\gamma$-ray emissions in inner galactic plane (IGP) with some areas have been masked (see 3.3) to reduce the contribution from those point or extended sources,

- Inner galactic plane: $25^{\circ}<l<100^{\circ},-5^{\circ}<b<5^{\circ}$

Fig.2 shows the map of masked IGP region, white spaces are masked regions due to the existence of some sources inside of them.

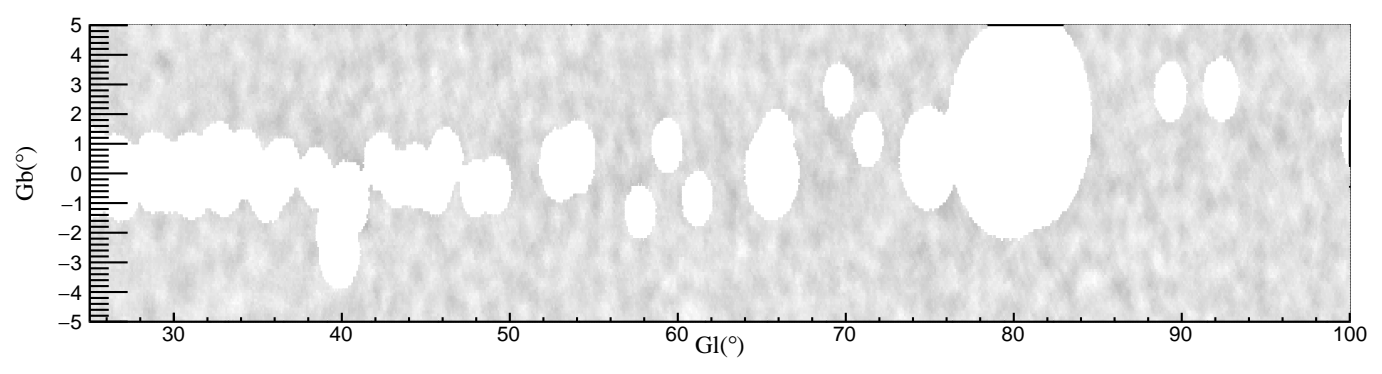

Figure 2: Map of IGP after extracting sources. White spaces indicate masked regions.

After background estimation, excess of the whole IGP region can be measured. Table 1 shows significance at each energy bin. For spectral energy distribution, the preliminary results is presented at the presentation.

\section{References}

[1] T. K. Gaisser, Cosmic rays and particle physics (Cambridge and New York, Cambridge University Press, 1990, 292 p., 1990).

[2] X. Bai, B. Y. Bi, X. J. Bi, Z. Cao, S. Z. Chen, Y. Chen, A. Chiavassa, X. H. Cui, Z. G. Dai, D. della Volpe, et al., arXiv e-prints arXiv:1905.02773 (2019), 1905.02773.

[3] D. Heck, J. Knapp, J. N. Capdevielle, G. Schatz, and T. Thouw, CORSIKA: a Monte Carlo code to simulate extensive air showers. (TIB Hannover, 1998).

[4] S. Chen, in 36th International Cosmic Ray Conference (ICRC2019) (2019), vol. 36 of International Cosmic Ray Conference, p. 219. 
[5] S. Agostinelli, J. Allison, K. Amako, J. Apostolakis, H. Araujo, P. Arce, M. Asai, D. Axen, S. Banerjee, G. Barrand, et al., Nuclear Instruments and Methods in Physics Research A 506, 250 (2003).

[6] F. Aharonian, Q. An, Axikegu, L. X. Bai, Y. X. Bai, Y. W. Bao, D. Bastieri, X. J. Bi, Y. J. Bi, H. Cai, et al., arXiv e-prints arXiv:2010.06205 (2020), 2010.06205.

[7] S. S. Wilks, Annals of Mathematical Statistics 9, 60 (1938).

\begin{tabular}{ccccc}
\hline \hline Energy $(\log (\mathrm{E} / \mathrm{TeV}))$ & $N_{\text {on }}$ & $N_{b}$ & $N_{s}$ & significance \\
\hline $1.0-1.2$ & 2291024 & 2281916.5 & 9107.5 & 6.03 \\
$1.2-1.4$ & 801892 & 796833.8 & 5058.2 & 5.66 \\
$1.4-1.6$ & 64240 & 62371.2 & 1868.8 & 7.45 \\
$1.6-1.8$ & 8367 & 7570.2 & 796.8 & 9.00 \\
$1.8-2.0$ & 4782 & 4426.7 & 355.3 & 5.27 \\
$2.0-2.2$ & 561 & 415.8 & 145.2 & 6.76 \\
$2.2-2.4$ & 133 & 55.4 & 77.6 & 8.81 \\
$2.4-2.6$ & 33 & 19.9 & 13.1 & 2.67 \\
$2.6-2.8$ & 21 & 4.4 & 16.6 & 5.71 \\
$2.8-3.0$ & 2 & 2.0 & 0.0 & 0.03 \\
\hline
\end{tabular}

Table 1: Measurements of GDE excess at each energy bin. $N_{o n}, N_{b}$ and $N_{s}$ represents sum of the event count, sum of the background count and excess of the region IGP respectively. The significance here is $\sqrt{T S}$. 


\section{Full Authors List: LHAASO Collaboration}

Zhen $\mathrm{Cao}^{1,2,3}$, F. Aharonian ${ }^{4,5}$, Q. An ${ }^{6,7}$, Axikegu ${ }^{8}$, L.X. Bai ${ }^{9}$, Y.X. Bai ${ }^{1,3}$, L.X. Bai ${ }^{9}$, Y.X. Bai ${ }^{1,3}$, Y.W. Bao ${ }^{10}$, D. Bastieri ${ }^{11}$, X.J. Bi ${ }^{1,2,3}$, Y.J. Bi ${ }^{1,3}$, H. Cai ${ }^{12}$, J.T. Cai ${ }^{11}$, Zhe Cao ${ }^{6,7}$, J. Chang ${ }^{13}$, J.F. Chang ${ }^{1,3,6}$, B.M. Chen ${ }^{14}$, E.S. Chen ${ }^{1,2,3}$, J. Chen ${ }^{9}$, Liang Chen $^{1,2,3}$, Liang Chen ${ }^{15}$, Long Chen ${ }^{8}$, M.J. Chen ${ }^{1,3}$, M.L. Chen ${ }^{1,3,6}$, Q.H. Chen ${ }^{8}$, S.H. Chen ${ }^{1,2,3}$, S.Z. Chen ${ }^{1,3}$, T.L. Chen ${ }^{16}$,X.L. $\mathrm{Chen}^{1,2,3}$, Y. Chen $^{10}$, N. Cheng ${ }^{1,3}$, Y.D. Cheng ${ }^{1,3}$, S.W. Cui ${ }^{14}$, X.H. Cuii ${ }^{17}$, Y.D. Cui ${ }^{18}$, B. D'Ettorre Piazzoli ${ }^{19}$, B.Z. Dai ${ }^{20}$, H.L. Dai $^{1,3,6}$, Z.G. Dai ${ }^{7}$, Danzengluobu ${ }^{16}$, D. della Volpe ${ }^{21}$, X.J. Dong ${ }^{1,3}$, K.K. Duan ${ }^{13}$, J.H. Fan ${ }^{11}$, Y.Z. Fan ${ }^{13}$, Z.X. Fan ${ }^{1,3}$, J. Fang ${ }^{20}$, K. Fang $^{1,3}$, C.F. Feng ${ }^{22}$, L. Feng ${ }^{13}$, S.H. Feng ${ }^{1,3}$, Y.L. Feng ${ }^{13}$, B. Gao ${ }^{1,3}$, C.D. Gao ${ }^{22}$, L.Q. Gao ${ }^{1,2,3}$, Q. Gao ${ }^{16}$, W. Gao ${ }^{22}$, M.M. Ge $^{20}$, L.S. Geng ${ }^{1,3}$, G.H. Gong ${ }^{23}$, Q.B. Gou ${ }^{1,3}$, M.H. Gu ${ }^{1,3,6}$, F.L. Guo ${ }^{15}$, J.G. Guo ${ }^{1,2,3}$, X.L. Guo ${ }^{8}$, Y.Q. Guo ${ }^{1,3}$, Y.Y. Guo ${ }^{1,2,3,13}$, Y.A. $\mathrm{Han}^{24}$, H.H. He $\mathrm{H}^{1,2,3}$, H.N. He ${ }^{13}$, J.C. He ${ }^{1,2,3}$, S.L. He ${ }^{11}$, X.B. He ${ }^{18}$, Y. He ${ }^{8}$, M. Heller ${ }^{21}$, Y.K. Hor ${ }^{18}$, C. Hou ${ }^{1,3}$, H.B. Hu ${ }^{1,2,3}$, S.

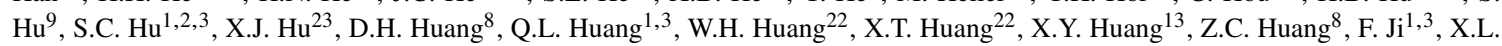
$\mathrm{Ji}^{1,3,6}$, H.Y. Jia ${ }^{8}$, K. Jiang ${ }^{6,7}$, Z.J. Jiang ${ }^{20}$, C. Jin ${ }^{1,2,3}$, T. Ke ${ }^{1,3}$, D. Kuleshov ${ }^{25}$, K. Levochkin ${ }^{25}$, B.B. Li ${ }^{14}$, Cheng Li ${ }^{6,7}$, Cong Li ${ }^{1,3}$,

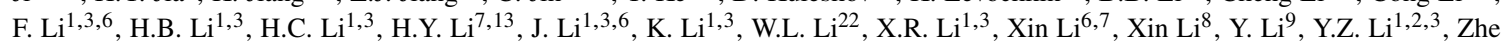

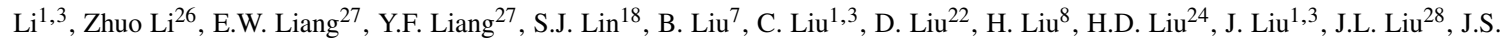

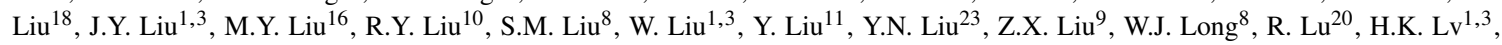

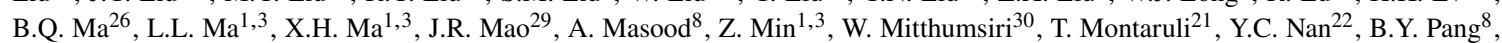
P. Pattarakijwanich ${ }^{30}$, Z.Y. Pei ${ }^{11}$, M.Y. Qi ${ }^{1,3}$, Y.Q. Qi ${ }^{14}$, B.Q. Qiao ${ }^{1,3}$, J.J. Qin ${ }^{7}$, D. Ruffolo ${ }^{30}$, V. Rulev ${ }^{25}$, A. Sáiz ${ }^{30}$, L. Shao ${ }^{14}$, O. Shchegolev $^{25,31}$, X.D. Sheng ${ }^{1,3}$, J.Y. Shi ${ }^{1,3}$, H.C. Song ${ }^{26}$, Yu.V. Stenkin ${ }^{25,31}$, V. Stepanov ${ }^{25}$, Y. Su ${ }^{32}$, Q.N. Sun ${ }^{8}$, X.N. Sun ${ }^{27}$, Z.B.

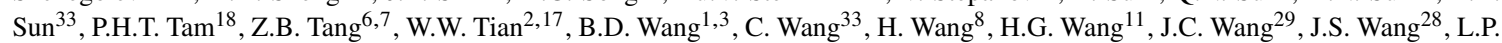
Wang $^{22}$, L.Y. Wang ${ }^{1,3}$, R.N. Wang ${ }^{8}$, W. Wang ${ }^{18}$, W. Wang ${ }^{12}$, X.G. Wang ${ }^{27}$, X.J. Wang ${ }^{1,3}$, X.Y. Wang ${ }^{10}$, Y. Wang ${ }^{8}$, Y.D. Wang ${ }^{1,3}$, Y.J. Wang $^{1,3}$, Y.P. Wang ${ }^{1,2,3}$, Z.H. Wang ${ }^{9}$, Z.X. Wang ${ }^{20}$, Zhen Wang ${ }^{28}$, Zheng Wang ${ }^{1,3,6}$, D.M. Wei $^{13}$, J.J. Wei ${ }^{13}$, Y.J. Wei ${ }^{1,2,3}$, T. Wen ${ }^{20}$, C.Y. $\mathrm{Wu}^{1,3}$, H.R. $\mathrm{Wu}^{1,3}$, S. Wu ${ }^{1,3}$, W.X. Wu ${ }^{8}$, X.F. Wu ${ }^{13}$, S.Q. Xi ${ }^{1,3}$, J. Xia ${ }^{7}, 13$, J.J. Xia ${ }^{8}$, G.M. Xiang ${ }^{2,15}$, D.X. Xiao ${ }^{16}$, G. Xiao ${ }^{1,3}$, H.B. Xiao ${ }^{11}$, G.G. Xin ${ }^{12}$, Y.L. Xin ${ }^{8}$, Y. Xing ${ }^{15}$, D.L. Xu ${ }^{28}$, R.X. Xu ${ }^{26}$, L. Xue ${ }^{22}$, D.H. Yan ${ }^{29}$, J.Z. Yan ${ }^{13}$, C.W. Yang 9 , F.F. Yang ${ }^{1,3,6}$, J.Y. Yang ${ }^{18}$, L.L. Yang ${ }^{18}$, M.J. Yang ${ }^{1,3}$, R.Z. Yang ${ }^{7}$, S.B. Yang ${ }^{20}$, Y.H. Yao ${ }^{9}$, Z.G. Yao ${ }^{1,3}$, Y.M. Ye ${ }^{23}$, L.Q. Yin ${ }^{1,3}$, N. Yin ${ }^{22}$, X.H. You $^{1,3}$, Z.Y. You ${ }^{1,2,3}$, Y.H. Yu ${ }^{22}$, Q. Yuan ${ }^{13}$, H.D. Zeng ${ }^{13}$, T.X. Zeng ${ }^{1,3,6}$, W. Zeng ${ }^{20}$, Z.K. Zeng ${ }^{1,2,3}$, M. Zha ${ }^{1,3}$, X.X. Zhai ${ }^{1,3}$, B.B. Zhang $^{10}$, H.M. Zhang ${ }^{10}$, H.Y. Zhang ${ }^{22}$, J.L. Zhang ${ }^{17}$, J.W. Zhang ${ }^{9}$, L.X. Zhang ${ }^{11}$, Li Zhang ${ }^{20}$, Lu Zhang ${ }^{14}$, P.F. Zhang ${ }^{20}$, P.P. Zhang ${ }^{14}$, R. Zhang ${ }^{7,13}$, S.R. Zhang ${ }^{14}$, S.S. Zhang ${ }^{1,3}$, X. Zhang ${ }^{10}$, X.P. Zhang ${ }^{1,3}$, Y.F. Zhang ${ }^{8}$, Y.L. Zhang ${ }^{1,3}$, Yi Zhang ${ }^{1,13}$, Yong Zhang ${ }^{1,3}$, B. $Z_{\text {Zhao }}^{8}$, J. Zhao ${ }^{1,3}$, L. Zhao ${ }^{6,7}$, L.Z. Zhao ${ }^{14}$, S.P. Zhao ${ }^{13,22}$, F. Zheng ${ }^{33}$, Y. Zheng ${ }^{8}$, B. Zhou ${ }^{1,3}$, H. Zhou ${ }^{28}$, J.N. Zhou ${ }^{15}$, P. Zhou ${ }^{10}$, R. Zhou $^{9}$, X.X. Zhou ${ }^{8}$, C.G. Zhu ${ }^{22}$, F.R. Zhu ${ }^{8}$, H. Zhu ${ }^{17}$, K.J. Zhu ${ }^{1,2,3,6}$ and X. Zuo ${ }^{1,3}$

${ }^{1}$ Key Laboratory of Particle Astrophyics \& Experimental Physics Division \& Computing Center, Institute of High Energy Physics, Chinese Academy of Sciences, 100049 Beijing, China.

${ }^{2}$ University of Chinese Academy of Sciences, 100049 Beijing, China.

${ }^{3}$ TIANFU Cosmic Ray Research Center, Chengdu, Sichuan, China.

${ }^{4}$ Dublin Institute for Advanced Studies, 31 Fitzwilliam Place, 2 Dublin, Ireland.

${ }^{5}$ Max-Planck-Institut for Nuclear Physics, P.O. Box 103980, 69029 Heidelberg, Germany.

${ }^{6}$ State Key Laboratory of Particle Detection and Electronics, China.

${ }^{7}$ University of Science and Technology of China, 230026 Hefei, Anhui, China.

${ }^{8}$ School of Physical Science and Technology \& School of Information Science and Technology, Southwest Jiaotong University, 610031 Chengdu, Sichuan, China.

${ }^{9}$ College of Physics, Sichuan University, 610065 Chengdu, Sichuan, China.

${ }^{10}$ School of Astronomy and Space Science, Nanjing University, 210023 Nanjing, Jiangsu, China.

${ }^{11}$ Center for Astrophysics, Guangzhou University, 510006 Guangzhou, Guangdong, China.

${ }^{12}$ School of Physics and Technology, Wuhan University, 430072 Wuhan, Hubei, China.

${ }^{13}$ Key Laboratory of Dark Matter and Space Astronomy, Purple Mountain Observatory, Chinese Academy of Sciences, 210023 Nanjing, Jiangsu, China.

${ }^{14}$ Hebei Normal University, 050024 Shijiazhuang, Hebei, China.

${ }^{15}$ Key Laboratory for Research in Galaxies and Cosmology, Shanghai Astronomical Observatory, Chinese Academy of Sciences, 200030 Shanghai, China.

${ }^{16}$ Key Laboratory of Cosmic Rays (Tibet University), Ministry of Education, 850000 Lhasa, Tibet, China.

${ }^{17}$ National Astronomical Observatories, Chinese Academy of Sciences, 100101 Beijing, China.

${ }^{18}$ School of Physics and Astronomy \& School of Physics (Guangzhou), Sun Yat-sen University, 519000 Zhuhai, Guangdong, China.

${ }^{19}$ Dipartimento di Fisica dell'Università di Napoli 'Federico II”, Complesso Universitario di Monte Sant’Angelo, via Cinthia, 80126 Napoli, Italy.

${ }^{20}$ School of Physics and Astronomy, Yunnan University, 650091 Kunming, Yunnan, China.

${ }^{21}$ D'epartement de Physique Nucl'eaire et Corpusculaire, Facult'e de Sciences, Universit'e de Gen 'eve, 24 Quai Ernest Ansermet, 1211

Geneva, Switzerland.

${ }^{22}$ Institute of Frontier and Interdisciplinary Science, Shandong University, 266237 Qingdao, Shandong, China.

${ }^{23}$ Department of Engineering Physics, Tsinghua University, 100084 Beijing, China.

${ }^{24}$ School of Physics and Microelectronics, Zhengzhou University, 450001 Zhengzhou, Henan, China.

${ }^{25}$ Institute for Nuclear Research of Russian Academy of Sciences, 117312 Moscow, Russia. 
${ }^{26}$ School of Physics, Peking University, 100871 Beijing, China.

${ }^{27}$ School of Physical Science and Technology, Guangxi University, 530004 Nanning, Guangxi, China.

${ }^{28}$ Tsung-Dao Lee Institute \& School of Physics and Astronomy, Shanghai Jiao Tong University, 200240 Shanghai, China.

${ }^{29}$ Yunnan Observatories, Chinese Academy of Sciences, 650216 Kunming, Yunnan, China.

${ }^{30}$ Department of Physics, Faculty of Science, Mahidol University, 10400 Bangkok, Thailand.

${ }^{31}$ Moscow Institute of Physics and Technology, 141700 Moscow, Russia.

${ }^{32}$ Key Laboratory of Radio Astronomy, Purple Mountain Observatory, Chinese Academy of Sciences, 210023 Nanjing, Jiangsu, China.

${ }^{33}$ National Space Science Center, Chinese Academy of Sciences, 100190 Beijing, China. 